\title{
HODOGRAPH METHOD OF NONLINEAR STRESS ANALYSIS OF THICK-WALLED CYLINDERS AND SPHERES, INCLUDING POROUS MATERIALS
}

\author{
M. A. Biot \\ Royal Academy of Belgium, Brussels, Belgium \\ (Received 23 October 1975; revised 8 April 1976)
}

\begin{abstract}
A new method is developed, analogous to a hodograph method, for the nonlinear finite strain and stress analysis of thick-walled cylinders and spheres under internal and external pressure. The problem is reduced to solving a single ordinary differential equation of the first order. It is applicable to plastic or elastic materials. The case of porous cylinder with fluid seepage is also discussed. The method provides drastic simplifications while remaining quite general and rigorous.
\end{abstract}

\section{INTRODUCTION}

A classical procedure of solving nonlinear ordinary differential equations with two unknowns has been used extensively in many applications, and is generally referred to as the hodograph method. In this procedure two simultaneous equatiosns with two unknown functions of time are replaced by a single first order differential equation from which the time is eliminated. In this equation one of the unknowns is considered solely as a function of the other which becomes the independent variable.

A similar procedure may be used in a completely general finite stress and deformation analysis with axial or spherical symmetry. In this case, there are two principal stresses to be evaluated, one in the radial direction and the other normal to this direction. In analogy with the hodograph method we have shown that an ordinary differential equation may be derived were the radial stress is the unknown and the other is the independent variable. The procedure was presented earlier [1] in the context of the stress analysis around cavities in rock. Our purpose here is to show how it is applicable to thick walled cylinders and spheres and provide further extensions of the theory for triaxial strain and porous materials.

The method is applicable to large strains and stresses and materials which are strongly nonlinear, whether plastic or elastic. Materials may be either isotropic or orthotropic in the radial direction.

A considerable advantage of the method results from the fact that the basic solution is given by a curve in a plane where the coordinates are the principal stresses. Since yielding or cracking conditions are usually expressed by a curve in the same plane, failure is determined very simply by the intersection of this curve and the solution curve. The usefulness and simplicity of this approach is well illustrated in previous work[1]. The name hodograph method finds its origin in a classical problem of nonlinear dynamics of a mass point, governed by the following equation

$$
\frac{\mathrm{d}^{2} x}{\mathrm{~d} t^{2}}=f\left(x, \frac{\mathrm{d} x}{\mathrm{~d} t}\right)
$$

where $x$ is the displacement as a function of the time $t$. It may be written in the form

$$
\begin{aligned}
& \frac{\mathrm{d} v}{\mathrm{~d} t}=f(x, v) \\
& \frac{\mathrm{d} x}{\mathrm{~d} t}=v
\end{aligned}
$$

The new variable $v$ is the velocity. Dividing the first equation by the second yields

$$
\frac{\mathrm{d} v}{\mathrm{~d} x}=\frac{v}{f(x, v)}
$$


This is a first order differential equation where $v$ is the unknown and $x$ the independent variable. The method used in the present analysis is a generalization to the case where the equations are of the type

$$
\begin{aligned}
& \frac{\mathrm{d} x}{\mathrm{~d} t}=\varphi(t) f_{1}(x, y) \\
& \frac{\mathrm{d} y}{\mathrm{~d} t}=\varphi(t) f_{2}(x, y) .
\end{aligned}
$$

By a procedure similar to the hodograph method we obtain the first order differential equation

$$
\frac{\mathrm{d} y}{\mathrm{~d} x}=\frac{f_{2}(x, y)}{f_{1}(x, y)}
$$

with a single unknown $y$ function of $x$. In the application to static problems of the present paper the equations are of the type (1.4) and the role of $t$ is played by the radial coordinate $r$ with $\varphi(r)=1 / r$.

\section{THICK-WALLED CYLINDER WITH FINITE PLANE STRAIN}

Finite deformation with axial symmetry is defined as follows. A material point initially at a distance $r$ from the axis is displaced by a finite amount $V$, along the same radial direction. The value of $V$ is assumed to be a function of $r$ only. At a displaced point the finite plane strain is represented by the principal component $\epsilon_{1}$ in the radial direction and the principal component $\epsilon_{2}$ in the circumferential direction. Their values are

$$
\begin{aligned}
\epsilon_{1} & =\frac{\mathrm{d} V}{\mathrm{~d} r} \\
\epsilon_{2} & =\frac{V}{r} .
\end{aligned}
$$

The corresponding principal stresses $\tau_{1}$ and $\tau_{2}$ respectively in the radial and circumferential directions are defined as normal forces acting at the coordinate $r+V$, per unit initial area of the medium. Positive values of $\tau_{1}$ and $\tau_{2}$ represent tensile stresses. The equilibrium condition for these stresses is obtained from the principle of virtual work

$$
2 \pi \int_{a}^{b}\left(\tau_{1} \delta \epsilon_{1}+\tau_{2} \delta \epsilon_{2}\right) r \mathrm{~d} r=0
$$

where $a$ and $b$ are the inner and outer initial radii of the cylinder. By varying $V$ with the definition (2.1) for $\epsilon_{1}$ and $\epsilon_{2}$ we derive

$$
\frac{\mathrm{d} \tau_{1}}{\mathrm{~d} r}+\frac{\tau_{1}-\tau_{2}}{r}=0
$$

On the other hand elimination of $V$ between eqns (2.1) leads to

$$
\frac{\mathrm{d} \epsilon_{2}}{\mathrm{~d} r}=\frac{\epsilon_{1}-\epsilon_{2}}{r}
$$

Finally elimination of $r$ between eqns (2.4) and (2.5) yields

$$
\frac{\mathrm{d} \tau_{1}}{\mathrm{~d} \epsilon_{2}}=-\frac{\tau_{1}-\tau_{2}}{\epsilon_{1}-\epsilon_{2}}
$$

We now assume that finite stress-strain relations in plane strain are known in the form 


$$
\begin{aligned}
& \epsilon_{1}=\epsilon_{1}\left(\tau_{1}, \tau_{2}\right) \\
& \epsilon_{2}=\epsilon_{2}\left(\tau_{1}, \tau_{2}\right) .
\end{aligned}
$$

They may represent finite elastic of plastic deformations. The material may be orthotropic provided the directions of symmetry are radial and circumferential. Substituting the values (2.7) of $\epsilon_{1}$ and $\epsilon_{2}$ into eqn (2.6) we obtain

$$
\frac{\mathrm{d} \tau_{1}}{\mathrm{~d} \tau_{2}}=-\frac{\partial \epsilon_{2} / \partial \tau_{2}}{\left[\left(\epsilon_{1}-\epsilon_{2}\right) /\left(\tau_{1}-\tau_{2}\right)\right]+\partial \epsilon_{2} / \partial \tau_{1}}
$$

Since the right side is a known function of $\tau_{1}$ and $\tau_{2}$, eqn (2.8) is a first order ordinary differential equation for $\tau_{1}$ as a function of $\tau_{2}$. Its integral is

$$
\tau_{2}=\psi(\tau, C)
$$

where $C$ is a constant of integration. Using the equilibrium eqn (2.4) we derive

$$
\int_{\tau_{a}}^{\tau_{1}} \frac{\mathrm{d} \tau_{1}}{\psi\left(\tau_{1}, C\right)-\tau_{1}}=\log \frac{r}{a}
$$

The value of $C$ is obtained from this equation by writing

$$
\int_{\tau_{a}}^{\tau_{b}} \frac{\mathrm{d} \tau_{1}}{\psi\left(\tau_{1}, C\right)-\tau_{1}}=\log \frac{b}{a}
$$

where $\tau_{a}$ and $\tau_{b}$ are the radial stresses at the inner and outer radii, $a$ and $b$.

Hence a complete solution is derived where $\tau_{1}, \tau_{2}$ and $V$ are obtained as functions of $r$. Note that $\tau_{1} \tau_{2}$ are the stresses at the displaced points of coordinates $r+V$.

The solution for the case where pressures per unit deformed area are given at the inner and outer radii as well as the stress-strain relations for isotropic or elastic materials has been discussed earlier [1].

A considerable advantage of the present solution is due to the fact that it is a curve in the $\tau_{1} \tau_{2}$ plane. Yield or fracture curves are also represented in the same plane so that intersection of the two curves yields the failure condition. This was discussed earlier[1] in the context of rock fracture.

The finite strain of a rubber membrane with a circular hole under axially symmetric stretching has been analyzed by deriving a first order differential equation with the two stretch ratios as variables [2], using the tensor invariants of isotropic elasticity. This special case may be solved immediately in the elementary and more general context of the hodograph method.

\section{THICK-WALLED CYLINDER WITH TRIAXIAL STRAIN}

The same eqns (2.1) and (2.4) are valid for radial and circumferential strains and for the equilibrium conditions for the stresses $\tau_{1}$ and $\tau_{2}$. The difference lies solely in the fact that the radial and circumferential strains $\epsilon_{1}$ and $\epsilon_{2}$ now depend also on the axial strain $\epsilon_{3}$. We write

$$
\begin{aligned}
& \epsilon_{1}=\epsilon_{1}\left(\tau_{1}, \tau_{2}, \epsilon_{3}\right) \\
& \epsilon_{2}=\epsilon_{2}\left(\tau_{1}, \tau_{2}, \epsilon_{3}\right) .
\end{aligned}
$$

If we assume the axial strain $\epsilon_{2}$ to be constant it plays the role of an unknown parameter and we may proceed exactly as in the previous case where $\epsilon_{2}=0$. The solution of the differential eqn (2.8) is now written

$$
\tau_{2}=\psi\left(\tau_{1}, C, \epsilon_{3}\right)
$$

with two unknown parameters $C$ and $\epsilon_{3}$. Two equations are available to determine these parameters. The first one is (2.11) which depends on the stresses at the circular boundaries. The 
other is provided by the expression for the total axial force $F$. It is obtained by expressing the local axial stress as

$$
\tau_{3}=\tau_{3}\left(\tau_{1}, \tau_{2}, \epsilon_{3}\right)
$$

The total axial force is then

$$
F\left(C, \epsilon_{3}\right)=2 \pi \int_{a}^{b} \tau_{3} r \mathrm{~d} r
$$

In this integral $\tau_{3}$ is expressed as a function of $r, C$ and $\epsilon_{3}$ using eqns (2.10), (3.2) and (3.3). Hence if we know the total axial force $F$ we may determine $C$ and $\epsilon_{3}$ from eqns (2.11) and (3.4). If the deformation is not large $F^{\prime}$ may be evaluated from the internal and external areas of the end plates of the cylinder as well as the internal and external pressures applied.

The stress-strain relations (3.1) and (3.3) are valid for orthotropic plastic materials with axes of symmetry oriented radially and circumferentially. For elastic materials it is easily shown by a procedure similar to that used previously [1]. That the functions must satisfy the conditions

$$
\begin{aligned}
& \frac{\partial \epsilon_{1}}{\partial \tau_{2}}=\frac{\partial \epsilon_{2}}{\partial \tau_{1}} \\
& \frac{\partial \tau_{3}}{\partial \tau_{1}}=-\frac{\partial \epsilon_{1}}{\partial \epsilon_{3}} \\
& \frac{\partial \tau_{3}}{\partial \tau_{2}}=-\frac{\partial \epsilon_{2}}{\partial \epsilon_{3}} .
\end{aligned}
$$

These relations are a consequence of the existence of an elastic potential.

If the material is isotropic the stress-strain relations are determined by a single function with the property

$$
\varphi\left(x_{1}, x_{2}, x_{3}\right)=\varphi\left(x_{1}, x_{3}, x_{2}\right)
$$

We may then write

$$
\begin{aligned}
& \epsilon_{1}=\varphi\left(\tau_{1}, \tau_{2}, \tau_{3}\right) \\
& \epsilon_{2}=\varphi\left(\tau_{2}, \tau_{3}, \tau_{1}\right) \\
& \epsilon_{3}=\varphi\left(\tau_{3}, \tau_{1}, \tau_{2}\right) .
\end{aligned}
$$

The last equation is solved for $\tau_{3}$. This value is then substituted in the values of $\epsilon_{1}$ and $\epsilon_{2}$, thus yielding eqns (3.1). For an elastic material the function $\varphi$ must satisfy the additional relation

$$
\frac{\partial \epsilon_{1}}{\partial \tau_{2}}=\frac{\partial \epsilon_{2}}{\partial \tau_{1}}
$$

\section{POROUS THICK-WALLED CYLINDER}

In many applications the cylinder wall is constituted by a porous material, such that when fluid pressure is applied internally a fluid flow takes place radially through the pores. For constant or approximately constant permeability, assuming negligible fluid compressibility and Darcy's law, the radial distribution of fluid pressure in steady state flow is

$$
p=A \log r+B
$$

where $A$ and $B$ are constants determined by the boundary conditions at the inner and outer wall. We shall also assume the deformation to be sufficiently small so that $p$ at $r$ and $r+V$ is approximately the same. These assumptions find their application in the fact that a large category of porous materials are strongly nonlinear already in a range not exceeding a $1 \%$ strain. Another applicable assumption is expressed by introducing effective stresses $\bar{\tau}_{1}, \bar{\tau}_{2}$. We write 


$$
\begin{aligned}
& \tau_{1}=\bar{\tau}_{1}-p \\
& \tau_{2}=\bar{\tau}_{2}-p .
\end{aligned}
$$

Since positive values of $\tau_{1}, \tau_{2}$ represent tensile stresses we see that the effective stresses $\bar{\tau}_{1}, \bar{\tau}_{2}$ are the stresses in excess above the hydrostatic fluid stress $-p$. For simplicity we shall assume plane strain deformations $\left(\epsilon_{3}=0\right)$. However the treatment may be extended to triaxial strain following the procedure of the previous section.

We first consider the stress-strain relations in plane strain for the case $p=0$. They are written

$$
\begin{aligned}
& \bar{\epsilon}_{1}=\bar{\epsilon}_{1}\left(\bar{\tau}_{1}, \bar{\tau}_{2}\right) \\
& \bar{\epsilon}_{2}=\bar{\epsilon}_{2}\left(\bar{\tau}_{1}, \bar{\tau}_{2}\right) .
\end{aligned}
$$

If we now apply a total hydrostatic pressure $p$ in the fluid and the solid matrix the strain becomes

$$
\begin{aligned}
& \epsilon_{1}=\bar{\epsilon}_{1}\left(\bar{\tau}_{1}, \bar{\tau}_{2}\right)-c p \\
& \epsilon_{2}=\bar{\epsilon}_{2}\left(\bar{\tau}_{1}, \bar{\tau}_{2}\right)-c p
\end{aligned}
$$

where $c$ is a plane strain compressibility. These relations embody the properties of semi-linearity already discussed earlier[3]. Strictly speaking of course eqns (4.5) embody a physical approximation which should not alter results significantly.

Substitution of the values (4.2) into the equilibrium eqns (2.4) and of the values (4.5) into eqns (2.5) yields

$$
\begin{aligned}
\frac{\mathrm{d} \tau_{1}}{\mathrm{~d} r}+\frac{\bar{\tau}_{1}-\bar{\tau}_{2}-A}{r} & =0 \\
\frac{\mathrm{d} \bar{\epsilon}_{2}}{\mathrm{~d} r}-\frac{\bar{\epsilon}_{1}-\bar{\epsilon}_{2}+c A}{r} & =0 .
\end{aligned}
$$

By eliminating $r$ between these two equations we derive

$$
\frac{\mathrm{d} \bar{\tau}_{1}}{\mathrm{~d} \bar{\epsilon}_{2}}=-\frac{\bar{\tau}_{1}-\bar{\tau}_{2}-A}{\bar{\epsilon}_{1}-\bar{\epsilon}_{2}-c A} .
$$

Hence the procedure of Section 2 for the non-porous material is valid in this case and the differential eqn $(2.8)$ is replaced by

$$
\frac{d \bar{\tau}_{1}}{\mathrm{~d} \bar{\tau}_{2}}=-\frac{\partial \bar{\epsilon}_{2} / \partial \bar{\tau}_{2}}{\left[\left(\bar{\epsilon}_{1}-\bar{\epsilon}_{2}+c A\right) /\left(\bar{\tau}_{1}-\bar{\tau}_{2}-A\right)\right]+\partial \bar{\epsilon}_{2} / \partial \bar{\tau}_{1}} .
$$

This is again an ordinary differential equation for $\bar{\tau}_{1}$, with $\bar{\tau}_{2}$ as independent variable. The case of isotropy and perfect elasticity is treated in the same manner as before [1]. The integral of (4.5) is a curve in the plane $\bar{\tau}_{1} \bar{\tau}_{2}$ of the effective stresses which provides a particular convenient tool for the prediction of material failure.

As an illustration consider the case of a porous cylinder subject to a pressure $p_{1}$ at its interior radius $r=a$ and a smaller pressure $p_{2}$ at its exterior radius $r=b$. The value of $A$ in this case is negative and equal to

$$
A=-\frac{p_{1}-p_{2}}{\log (b / a)}
$$

In the plane $\bar{\tau}_{1} \bar{\tau}_{2}$ the slope of the integral is equal to zero on the straight isocline

$$
\bar{\tau}_{1}-\bar{\tau}_{2}-A=0
$$

which is represented in Fig. 1. At both $r=a$ and $r=b$ the effective radial stress $\bar{\tau}_{1}$ is zero. A 


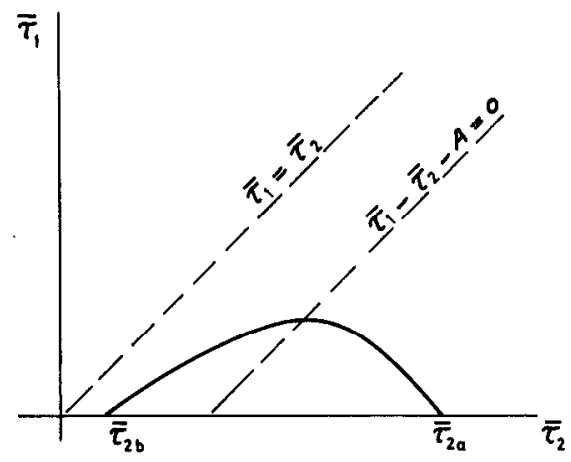

Fig. 1. Integral curve $\bar{\tau}_{1}$ vs $\bar{\tau}_{2}$ for a porous cylinder subject to internal pressure $p_{1}$ and external pressure $p_{2}\left(p_{1}>p_{2}\right)$. Circumferential effective stresses are $\bar{\tau}_{2 a}$ and $\bar{\tau}_{2 b}$ at inner and outer radius respectively.

brief examination of the other isoclines shows that the integral of eqn (4.8) must resemble the curve shown in Fig. 1 . The value of the radial effective stress $\bar{\tau}_{1}$ is maximum on the straight line (4.10).

\section{THICK-WALLED SPHERE}

The case of spherical symmetry was also analyzed in the earlier work [1]. The principal stress and strain in the radial direction are $\tau_{1}$ and $\epsilon_{1}$. In any direction normal to a radius the stress and strain are $\tau_{2}$ and $\epsilon_{2}$. The stresses are again per unit initial area and the finite radial displacement is $V$. By the principle of virtual work the stress equilibrium equation is found to be

$$
\frac{\mathrm{d} \tau_{1}}{\mathrm{~d} r}+\frac{2\left(\tau_{1}-\tau_{2}\right)}{r}=0
$$

Equations (2.1) for the strain remain the same and the stress strain relations are written

$$
\begin{aligned}
& \epsilon_{1}=\epsilon_{1}\left(\tau_{1}, \tau_{2}\right) \\
& \epsilon_{2}=\epsilon_{2}\left(\tau_{1}, \tau_{2}\right) .
\end{aligned}
$$

Note that in this case the material is assumed to be transverse isotropic with the axis of symmetry along a radius, and the stress $\tau_{2}$ applied isotropically around this axis. The differential eqn (2.8) now becomes

$$
\frac{\mathrm{d} \tau_{1}}{\mathrm{~d} \tau_{2}}=-\frac{2 \partial \epsilon_{2} / \partial \tau_{2}}{\left[\left(\epsilon_{1}-\epsilon_{2}\right) /\left(\tau_{1}-\tau_{2}\right)\right]+2 \partial \epsilon_{2} / \partial \tau_{1}}
$$

The material may be plastic or elastic. The case of isotropic as well as elastic materials has been discussed [1]. A simple quadrature analogous to (2.10) is obtained from the equilibrium eqn (5.1) and yields the initial coordinate $r$ as a function $\tau_{1}$.

\section{REFERENCES}

1. M. A. Biot, Exact simplified non-linear stress and fracture analysis around cavities in rock. Int. J. Rock. Mech. Min. Sci. and Geomech. 11, 261-266 (1974).

2. W. H. Yang, Stress concentration in a rubber sheet under axially symmetric stretching. J. Appl. Mech. 942-946 (Dec. 1967).

3. M. A. Biot, Nonlinear and semilinear rheology of porous solids. J. Geoph. Res. 78, 4924-4937 (1973). 